\title{
UTILIZACION DE TRES TIPOS DE ABONO ORGANICO EN LA CRIANZA Y REPRODUCCION DE LA LOMBRIZ Eisenia foetida EN SAN MARTíN
}

Gilberto U. Ascón Dionicio *

\section{RESUMEN}

El presente trabajo se realizó en la Estación Experimental El "Porvenir" INIA-San Martín, distrito de J uan Guerra, provincia y Región San Martín en el período diciembre de 1991 a noviembre de 1992; utilizando 3 tratamientos con 2 repeticiones para cada uno.

T1 : Lombriz E. foetida + estiércol de ganado vacuno + paja de arroz.

T2 : Lombriz E. foetida + estiércol de gallinas de postura + paja de arroz.

T3: $\quad$ Lombriz E. foetida + estiércol de cuy + paja de arroz.

La importancia del presente estudio radica en haber determinado la crianza y reproducción de la lombriz Eisenia foetida, cuando se utilizó 3 tipos de abono orgánico en la preparación de su alimento en una proporción de 3 de estiércol por 1 de paja de arroz.

La mayor cantidad de lombrices fue en el tratamiento T3. Sin embargo el humus de mayor calidad se obtuvo con el tratamiento T1.

\section{INTRODUCCION}

La lombricultura se inició en 1950 en EE.UU. con una especie conocida como la lombriz roja, siendo su verdadero nombre científico Eisenia foetida, Ferruzzi, C. (1987). Desde entonces se han realizado estudios que han tenido como resultados varios tipos de lombrices rojas cada vez más selectas, pero que en la actualidad los tipos más utilizados en la lombricultura son tres: Eisenia foetida, Lombricus rubellus y el rojo híbrido; de éstas la más difundida en el mundo es la lombriz Eisenia foetida, pertenece al phylum Anélidos clase Oligoquetos, explotada a

\footnotetext{
* Biólogo Pesquero investigador CRI-IIAP-San Martín. A partado 37 Tarapoto - Perú
} 
nivel industrial por países como EE.UU., J apón, Italia, España y Chile principalmente.

En el Perú la lombricultura se inició hace una década y se fue expandiendo a nivel nacional en los 5 últimos años en forma muy paulatina. En la Región San Martín podemos decir que es una actividad reciente.

La lombriz E. foetida, es una de las especies más versátiles para su producción o explotación en cautividad; Ascón, G. (1993).

Al estado adulto mide de $6-8 \mathrm{~cm}$. y su diámetro oscila de 3 $5 \mathrm{~mm}$., es de color rojo oscuro, respira a través de la piel y no tiene dientes, es hermafrodita insuficiente (necesita aparearse para reproducirse) la fecundación se realiza a través del clítelo, es extraordinariamente prolífica madura a los 90 días de nacida, su peso es aproximadamente $1.0 \mathrm{~g}$. y su promedio de vida en un criadero es de 12 15 años.

Silva, F. (1985), afirma que en Australia y Nueva Zelandia se ha demostrado que $\mathrm{E}$. foetida es una especie eurífaga, es decir, se ubica dentro de los animales de más amplio rango alimentario, desde los detritos orgánicos vegetales (rastrojo, residuos de hortalizas, frutos, malezas, etc.) hasta los coprolitos animales (estiércol de vacuno, oveja, conejo, cuy, etc.), son resistentes al estrés como variaciones de To, $\mathrm{pH}$ y humedad.

La lombricultura puede orientarse a la producción de carne de lombriz, la que perfectamente puede utilizarse en la alimentación animal directamente $o$ en harina para alimentos concentrados, o ser orientado a la producción de humus $100 \%$ orgánico y que puede ser utilizado como un excelente fertilizante en el cultivo de todo tipo de plantas vegetales.

Son estas las razones que nos condujeron a ejecutar el presente estudio con la finalidad de determinar la crianza y reproducción de la lombriz Eisenia foetida, cuando se utilizó tres tipos de abono orgánico en la preparación de su alimento.

\section{MATERIAL Y METODOS}

El presente estudio de investigación se ejecutó en la Estación Experimental El Porvenir-INIA-San Martín, ubicada a $14 \mathrm{~km}$ - Carretera Marginal Tarapoto - J uanjuí, distrito de J uan Guerra y provincia de San Martín, en el período diciembre de 1991 a noviembre de 1992 en 2 etapas: 


\section{PRIMERA ETAPA}

Esta etapa se realizó en el período comprendido entre diciembre de 1991 a junio de 1992 teniendo como finalidad adaptar la tecnología sobre la crianza de la lombriz Eisenia foetida generada en el IIAP de Pucallpa a la zona de la Región San Martín.

El trabajo se inició con la ubicación del terreno con una pendiente de $3 \%-5 \%$ y un cerco perimetral de $40 \mathrm{~m}$ por lado, con un área de $1600 \mathrm{~m}^{2}$. En esta área se construyeron 2 lechos de $1 \mathrm{~m}^{2}$ cada uno donde se realizó la adaptación y reproducción de la lombriz Eisenia foetida, estos lechos fueron cubiertos con tinglados de hojas de palma utilizando madera de la zona en forma de una cumba a dos aguas de $1.80 \mathrm{~m}$. de altura entre el piso del lecho y la cumba; $10 \mathrm{~m}$. de largo por $3 \mathrm{~m}$. de ancho.

En la preparación de alimento para las lombrices se utilizó estiércol de ganado vacuno y paja de arroz, dichas lombrices fueron donadas por el CRI-IIAP-SM en una cantidad de 2,000 lombrices todas adultas.

\section{SEGUNDA ETAPA}

Esta etapa se realizó en el período comprendido entre julio y noviembre de 1992 y consistió en determinar el mejor tipo de abono orgánico en la crianza y reproducción de la lombriz Eisenia foetida. Para lo cual se hizo un experimento aplicándose el diseño experimental en Bloques Completos al Azar ensayándose los tratamientos siguientes con 3 réplicas cada uno.

T1 : Lombriz E. foetida + estiércol de ganado vacuno + paja de arroz.

T2 : Lombriz E. foetida + estiércol de gallinas de postura + paja de arroz.

T3 : Lombriz E. foetida + estiércol de cuy + paja de arroz.

El desarrollo de este experimento tuvo las acciones siguientes:

Instalaciones de lechos o camas

Teniendo en cuenta el diseño experimental con 3 tratamientos y 3 repeticiones cada uno, se construyeron 9 lechos de $0.60 \mathrm{~m}$. de ancho por $1.0 \mathrm{~m}$. de largo utilizando tablas de $0.30 \mathrm{~m}$. de alto y 1 " de grosor. 
Preparación de alimento para lombrices

Se utilizó estiércoles de ganado vacuno, gallinas de postura, de cuyes y paja de arroz; la mezcla se hizo en una proporción por volumen, de 3 estiércol por 1 de paja ( $75 \%$ de estiércol por $25 \%$ de paja), colocándose una capa de paja de arroz luego una capa de estiércol y así sucesivamente hasta 80 ó $90 \mathrm{~cm}$. de altura, luego se procedió a regar todos los días y removerlo cada 24 ó 48 horas hasta que el alimento presentara un $\mathrm{pH}$ neutro y una humedad de $70 \%-80 \%$.

Siembra y alimentación en los lechos

La siembra se hizo luego de tener los lechos construidos y el alimento previamente preparado; este alimento se extendió sobre la base de los lechos; luego se remojó la superficie del sustrato y en seguida se colocaron las lombrices en las primeras horas de la mañana. Antes de colocar el alimento en los lechos se hizo la prueba de acidez o de supervivencia de la lombriz recomendado por Ferruzzi, C. (1987), que consistió en colocar 20 lombrices adultas en $1 \mathrm{~kg}$. de alimento luego se evaluaron a las 24,48 y 72 horas.

Frecuencia de postura y número de lombrices por cápsula

Para averiguar estos parámetros se tomó 3 recipientes pequeños de plástico en los cuales se colocó $1 \mathrm{~kg}$. de alimento y una pareja de lombrices apareados por tratamiento, esto se realizó al inicio, a mitad y al final del experimento.

Producción de lombrices y humus

Para determinar la cantidad de lombrices al final del experimento se hizo por conteo directo de cada uno de los lechos. Asímismo la producción de humus se hizo pesando el total de este fertilizante de cada uno de los lechos; la mortalidad de las lombrices fue por la presencia de enemigos depredadores.

Se hicieron análisis químicos de humus de cada tratamiento, se aplicó el Análisis de Varianza a los datos sobre producción de humus como a los elementos: nitrógeno, fósforo y potasio de los análisis de humus, con una prueba de Dunkan al 5\% según Arroyo, R. (1984).

Se tomaron registros de temperatura, $\mathrm{pH}$ y humedad 2 veces por semana de cada uno de los lechos. 
$\underline{\text { Utensilios utilizados }}$

01 carretilla tipo buggi

01 rastrillo con mango largo y puntas romas

01 azada

01 manguera de $3 / 4 "$

01 medidor de humedad de $0 \%-100 \%$ - Forestry - suppliers

01 peachímetro NIE - Co - product (NICUWKOOP - B.U.)

01 termómetro de $-10^{\circ} \mathrm{C}$ a $110^{\circ} \mathrm{C}$

16 metros de malla metálica

01 tamizador de humus

01 balanza tipo berkel

01 romana de 25 libras

01 wincha de $3 \mathrm{~m}$.

palas, pico, machetes, serrucho, martillo, cordel, regaderas, baldes y recipientes plástico de 1 litro.

\section{RESULTADOS}

Los resultados obtenidos en la primera etapa se presentan en el Cuadro 1.

\section{Cuadro 1}

Crianza y reproducción de la lombriz E. foetida

\begin{tabular}{|c|c|c|c|c|c|c||}
\hline $\begin{array}{c}\text { Lechos } \\
(\mathrm{N} N)\end{array}$ & $\begin{array}{c}\text { Inoculación } \\
\text { (cantidad) }\end{array}$ & $\begin{array}{c}\text { Proporción de } \\
\text { mezcla }\end{array}$ & $\begin{array}{c}\text { Tamaño de } \\
\text { lechos (m.) }\end{array}$ & $\begin{array}{c}\text { Lombrices } \\
\text { Adultos (№) }\end{array}$ & $\begin{array}{c}\text { Lombrices } \\
\text { Pequeños } \\
\text { (№) }\end{array}$ & $\begin{array}{c}\text { Tiempo de } \\
\text { crianza } \\
\text { (meses) }\end{array}$ \\
\hline 1 & 1,000 & $\begin{array}{c}4 \text { estiércol } \\
1 \text { de paja }\end{array}$ & $1.0 \times 1.0 \times 0.3$ & 1,800 & 1,400 & 4 \\
\hline 2 & 1,000 & $\begin{array}{c}3 \text { estiércol } \\
1 \text { de paja }\end{array}$ & $1.0 \times 1.0 \times 0.3$ & 3,600 & 2,820 & 4 \\
\hline
\end{tabular}


Como se puede observar en el cuadro anterior los mejores resultados se obtuvieron en el lecho № 2 con un total de 6,500 lombrices entre adultas y pequeñas sin considerar las cápsulas.

La segunda etapa que consistió en la determinación del mejor tipo de abono orgánico, para la preparación de alimento de las lombrices se utilizó una proporción en volumen de 3 de estiércol por 1 de paja de arroz $(75 \%$ de estiércol $+25 \%$ de paja) el cual dio mejores resultados en la primera etapa.

El tiempo que demoró el alimento en estar apto para ser utilizado fue de 45 días para los tratamientos T1 y T3 y de 75 días para el T2. La prueba de acidez o de supervivencia de la lombriz P20L se evaluó cada 24,48 y 72 horas. Esta prueba se realizó cada vez que se preparaba el alimento.

La alimentación que se le proporcionó a las lombrices se hizo de acuerdo al consumo del alimento en cada uno de los lechos.

Se demostró que la frecuencia de postura fue de 4 a 7 días y el número de lombrices fue de 1 a 2 lombricillas por cápsula.

Los resultados sobre la cantidad o producción de lombrices se puede apreciar en el Cuadro 2.

\section{Cuadro 2}

Cantidad de lombrices al final del experimento por tratamiento.

\begin{tabular}{||c|c|c|c|c||}
\hline \hline TRATAMIENTOS & REPETICIONES & $\begin{array}{c}\text { LOM BRICES } \\
\text { (№) }\end{array}$ & $\begin{array}{c}\text { CAPSULAS } \\
\text { (№) }\end{array}$ & $\begin{array}{c}\text { TOTAL (No DE } \\
\text { LOB. + CAPS.) }\end{array}$ \\
\hline T1 & 3 & 19,000 & 11,510 & 31,370 \\
\hline T2 & 3 & 7,630 & 1,177 & 8,807 \\
\hline T3 & 3 & 32,435 & 3,020 & 36,055 \\
\hline
\end{tabular}

Sobre la producción y análisis químico del humus lo podemos ver en los Cuadros 3 y 4. 


\section{Cuadro 3}

\section{Producción de humus al final del experimento}

\begin{tabular}{||c|c|r|r|r||}
\hline \hline TRATAMIENTOS & REPETICIONES & $\begin{array}{c}\text { HUMUS TAMIZ } \\
(\mathrm{Kg})\end{array}$ & $\begin{array}{c}\text { HUMUS } \\
\text { RESIDUAL (Kg) }\end{array}$ & $\begin{array}{c}\text { TOTAL } \\
(\mathrm{Kg})\end{array}$ \\
\hline T1 & 3 & 225 & 17.5 & 242.5 \\
\hline T2 & 3 & 150 & 24.5 & 174.5 \\
\hline T3 & 3 & 325 & 30 & 355 \\
\hline
\end{tabular}

\section{Cuadro 4}

\section{Análisis Químico de Humus por Tratamiento}

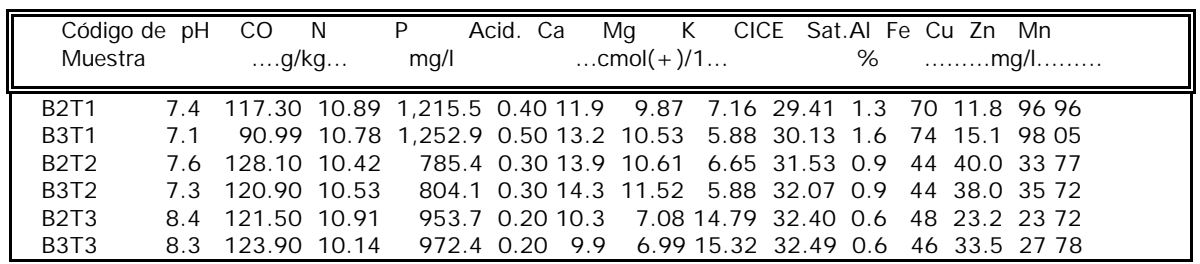

$\mathrm{B}=$ Bloques $\quad$ Metodología $=\mathrm{pH}$ : suelo/agua $1: 2.5 \mathrm{P}, \mathrm{kg}$

$\mathrm{T}=$ Tratamientos Acid, Ca y mg.kel $1 \mathrm{~N}$ N. total : KJ ELDAAL
MICROEL.NaHCo3 0.5N.EDTA-Superfloc $\mathrm{CO}$ : Nelson y Sammers

$\mathrm{N}, \mathrm{Ca}$, mg y Microel (Extracto) Absorción Atómica

Realizado: Laboratorio INIA-NCSU

Estación Experimental "SAN RAMON" Yurimaguas. 
Para la contrastación de la hipótesis se aplicó el Análisis de Varianza a la producción de humus y el Análisis de Varianza Factorial con una prueba de Duncan al $5 \%$ al N.P.K. del análisis químico del humus, como se puede apreciar a continuación:

\section{TABLA DE ANALISIS DE VARIANZA}

\begin{tabular}{|l|c|r|r|r|c|}
\hline $\begin{array}{l}\text { Fuente de } \\
\text { Variación }\end{array}$ & $\begin{array}{c}\text { Grados de } \\
\text { Libertadd }\end{array}$ & $\begin{array}{c}\text { Suma de } \\
\text { Cuadrados }\end{array}$ & $\begin{array}{c}\text { Cuadrados } \\
\text { Medios }\end{array}$ & $\begin{array}{c}\text { Valores de } \\
\text { F }\end{array}$ & Probab. \\
\hline Bloque & 2 & 912.50 & 456.250 & 0.85 & 0.4936 \\
Tratamiento & 4 & $5,535.17$ & $2,769.583$ & 5.14 & ns \\
Error & 4 & $2,155.33$ & 538.833 & & 0.0785 \\
No & 1 & $1,492.01$ & $1,492.010$ & 6.71 & ns \\
Aditividad & 3 & 663.32 & 221.108 & & \\
Residual & & $8,603.00$ & & & \\
\hline \multicolumn{1}{|c|}{ Total } & 8 & & & & \\
\hline
\end{tabular}

Coeficiente de variación $=27.04 \%$

TABLA DE ANALISIS DE VARIANZA FACTORIAL

\begin{tabular}{|l|c|r|r|r|r|}
\hline $\begin{array}{l}\text { Fuente de } \\
\text { Variación }\end{array}$ & $\begin{array}{c}\text { Grados de } \\
\text { Libertad }\end{array}$ & $\begin{array}{l}\text { Suma de } \\
\text { Cuadrados }\end{array}$ & \multicolumn{1}{l|}{$\begin{array}{l}\text { Cuadrados } \\
\text { Medios }\end{array}$} & $\begin{array}{l}\text { Valores } \\
\text { de F }\end{array}$ & Probab. \\
\hline Replicación & 1 & 292.094 & 292.094 & 16.6619 & $0.0551^{*}$ \\
Factor A & 2 & $\begin{array}{r}65370.079 \\
35.061\end{array}$ & $\begin{array}{r}32685.039 \\
17.531\end{array}$ & 1864.4526 & $0.0005^{* *}$ \\
Error & 2 & 3899741.701 & 1949870.851 & 16170.7817 & $0.0006^{* *}$ \\
Factor B & 2 & 131372.543 & 32843.136 & 272.3766 & $0.0003^{* *}$ \\
AB & 4 & 723.479 & 120.580 & & \\
Error & 6 & 4097534.957 & & & \\
\hline TOTAL & 17 & & & & \\
& & & & & \\
\hline
\end{tabular}

* Significativo

** Muy significativo. 
Prueba de Amplitud múltiple de Duncan

Valores de LSD $=10.40$

$S x=1.709$ al 0.050

ORDEN ORIGINAL

MEDIA $1=417.2 \mathrm{~A}$

MEDIA $2=270.5 \mathrm{C}$

MEDIA $3=329.6$ B

\section{ORDEN DE RANGO}

MEDIA $1=417.2 \mathrm{~A}$

MEDIA $3=329.6$ B

MEDIA $2=270.5 \mathrm{C}$

Asímismo por observación directa se notó algunos depredadores de la lombriz E. foetida como: sapos, hormigas, ciempiés y un turbelario carnívoro de vida libre perteneciente al género Bipalium sp.

Los resultados sobre humedad, $\mathrm{pH}$, temperatura se puede apreciar en las figuras 1,2 y 3 . 
FIG. I-2, REGISTROS PROMEDIOS DEL PORCENTAJE DE HUMEDAD Y PH POR TRATAMIENTO DURANTE EL EXPERIMENTO.
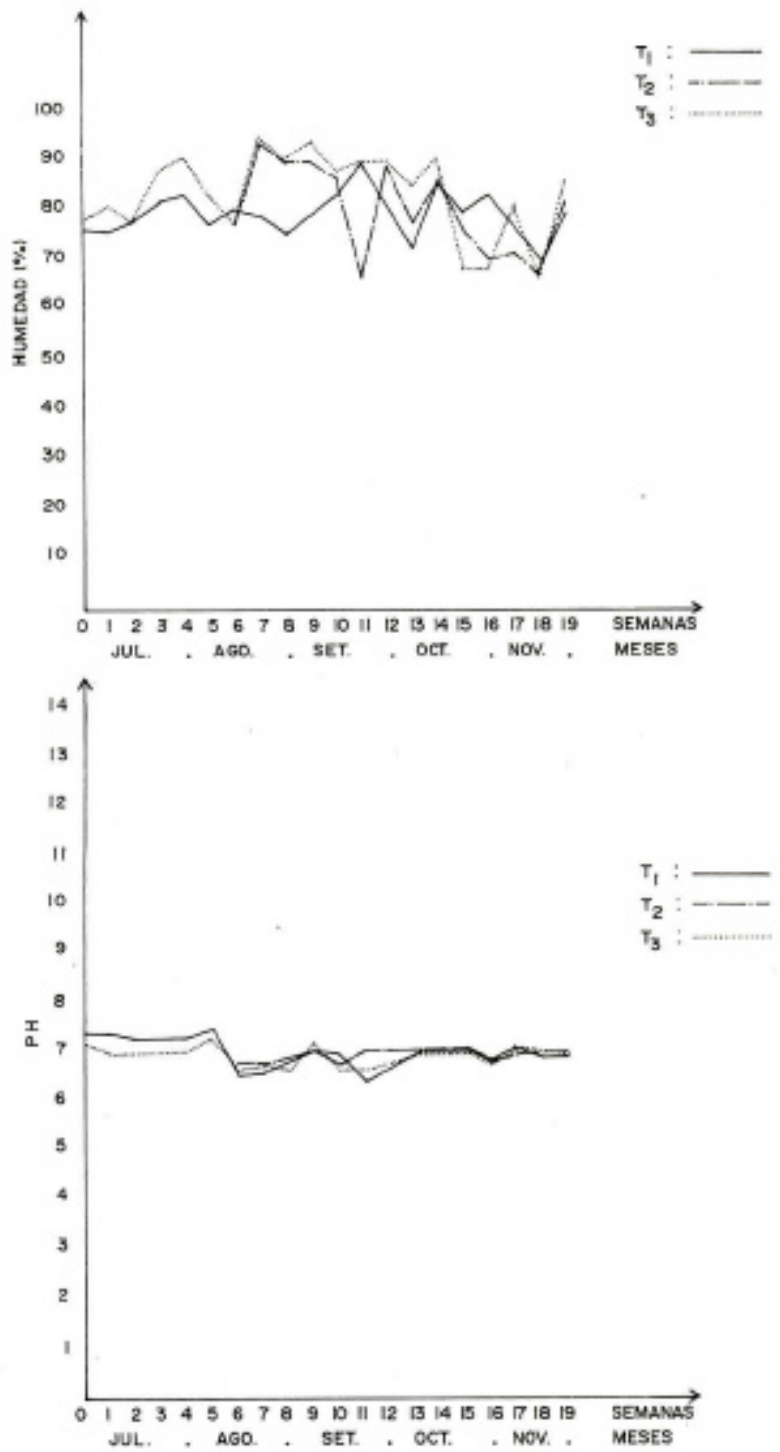
FIG. 3 REGISTROS PROMEDIOS DE TEMPERATURA POR TRATAMIENTO DURANTE EL EXPERIMENTO.

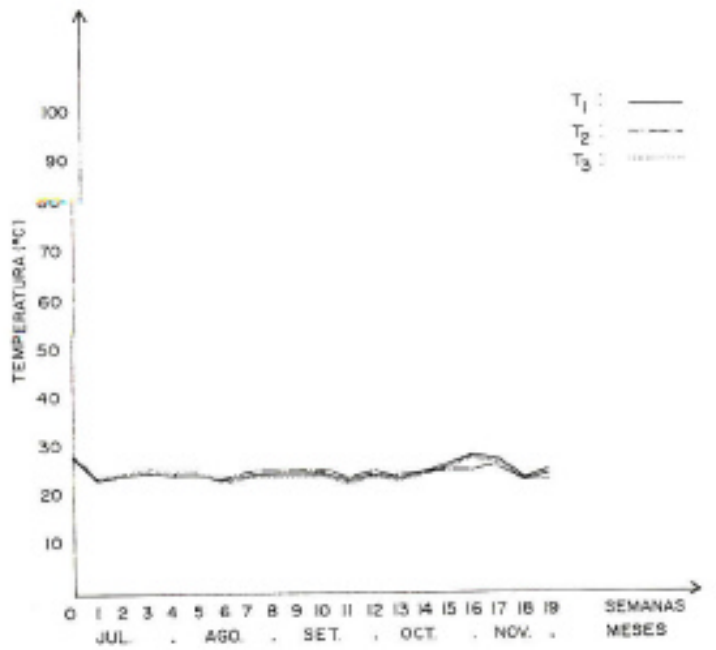




\section{DISCUSION}

Los resultados obtenidos en la primera etapa, sugieren que para la preparación del alimento de las lombrices especie Eisenia foetida debe ser de una proporción en volumen de 3 de estiércol por 1 de paja de arroz. Esta misma proporción lo recomienda Ferruzzi, C. (1987).

Siendo la proporción de 3:1 la que dio mejores resultados en la primera etapa, esta misma proporción se utilizó para la preparación del alimento de las lombrices en la segunda etapa; donde el tiempo necesario para que el alimento esté listo para ser utilizado fue de 30 días para los tratamientos T1 y T3 y de 75 días para el tratamiento T2. Esta diferencia se debió a que el tratamiento T2 (gallinaza) fue casi exento de celulosa y más bien con residuos de algunos insumos utilizados en la alimentación de las aves de postura, como: maíz, harina de pescado, así como la orina de las aves y las elevadas temperaturas determinó que presentara un $\mathrm{pH}$ muy ácido y el tiempo en descomponerse sea mayor. Estas observaciones concuerdan con lo manifestado por Ferruzzi, C. (1987), quien afirma que los estiércoles de gallinas, pollos, pavos y de aves en general no son aconsejables debido a su fuerte acidez, ocasionada por la elevada temperatura de fermentación $\left(90^{\circ} \mathrm{C}\right.$ ) y el prolongado espacio de tiempo para poder obtener un valor de $\mathrm{pH} 7.0$.

Para estar seguros si el alimento preparado estaba listo para ser utilizado en los lechos se hizo una prueba de acidez o prueba de sustrato realizado con 20 lombrices; conocidos por algunos autores como prueba de supervivencia de la lombriz y realizada con 50 lombrices, Aliaga, L. (1990) y Ferruzzi, C. (1987).

Según los resultados la alimentación que se le proporcionó a las lombrices fue de acuerdo al consumo del alimento en cada uno de los lechos y no con una periodicidad determinada como lo manifiesta Aliaga, L. (1990).

En cuanto a la frecuencia de postura y el número de lombricillas por cápsulas; nuestros resultados son similares a los obtenidos por la mayoría de autores donde la frecuencia de postura es de 7 días con 1 a 2 lombricillas por cápsula.

No obstante haber mortalidad por algunos depredadores, la cantidad de lombrices incluyendo cápsulas al final del experimento nos indica que con el tratamiento T3 se obtuvo una producción promedio mayor que los tratamientos T1 y T2. Lo que demuestra que para mayor proliferación o reproducción de la lombriz E. foetida es el tratamiento T3.

La frecuencia de postura, el número de lombricillas por cápsula y la cantidad de lombrices obtenidas nos demuestra que la especie E. foetida es muy prolífica. Silva, F. (1985) y Aliaga, L. (1990) manifiestan que un lecho de $1 \mathrm{~m}^{2}$ puede soportar densidades de hasta 50,000 lombrices y que 2 lombrices adultas pueden producir hasta 3,000 
lombrices por año en 5 generaciones.

Si observamos los resultados sobre la producción de humus por tratamiento vemos que no existen diferencias significativas entre bloques cuando se le aplicó un tratamiento estadístico de Análisis de Varianza al $5 \%$ según Arroyo, R. (1984). Mientras que cuando se aplicó un análisis de varianza factorial a los elementos nitrógeno, fósforo y potasio (N.P.K.) de los análisis químicos de humus por tratamiento, podemos apreciar que éstos son diferentes y por lo tanto influyen en el rendimiento, existiendo diferencias muy significativas entre los elementos N.P.K. por tratamiento al 5\%. Al aplicar la prueba de amplitud múltiple de Duncan se determinó que el mejor tratamiento en N.P.K. (calidad de humus) fue el tratamiento T1 como se muestra en los resultados.

Los resultados de humedad, Figura 1 , nos muestra que existen ciertas variaciones en los 3 tratamientos, es decir, no fue uniforme, concordando con lo que manifiesta Ferruzzi, C. (1987), no es fácil describir cual debe ser el nivel o porcentaje de humedad que debe tener un lecho. Para los americanos el nivel óptimo es $82.5 \%$, mientras que para otros autores como Aliaga, L. (1990) la humedad debe ser de $65 \%$ a $75 \%$.

Referente al pH Figura 2, se puede apreciar que existe una tendencia homogénea en los 3 tratamientos, registrándose valores de $\mathrm{pH}$ neutros, ligeramente ácidos o ligeramente alcalinos, siendo estos valores óptimos para la adaptación y reproducción de la lombriz Eisenia foetida.

La temperatura Figura 3, también mostró una tendencia casi uniforme en todo el proceso del experimento. Catalán, J. (1969) citado por Campoverde, L. (1977) manifiesta que la temperatura juega un papel importante en la solubilidad de los gases y sales así como en las reacciones biológicas y diversos fenómenos en un determinado sustrato. En nuestro caso se trató de uniformizar la temperatura protegiendo a los lechos con un cobertizo techado con hojas de palma.

\section{CONCLUSIONES}

1. Los resultados sugieren que para la preparación de alimento de la lombriz Eisenia foetida debe ser de una proporción en volumen de 3 de estiércol por 1 de paja de arroz con los tres tipos de abono orgánico.

2. Que el mejor tipo de abono orgánico para la reproducción y crianza de la lombriz E. foetida fue el tratamiento T3 (estiércol de cuy + paja de arroz) pero que para la calidad de humus fue el tratamiento T1 el que dio mejores resultados. 
3. Que uno de los depredadores más agresivos fue el turbelario carnívoro de vida libre perteneciente al género Bipalium sp.

\section{AGRADECIMIENTO}

Se agradece a las instituciones en las cuales el presente Estudio ha sido realizado, como parte de un convenio entre el Centro Regional de Investigación del IIAP-San Martín (CRI-IIAP-SM) y el Instituto de Investigación Agraria (INIA-SM). A la Red de Investigación de la Amazonía Peruana (RINAP) por su financiamiento. Asímismo a la Estación "San Roque" - PICT por los análisis de humus y al Licenciado en Estadística Marco Gálvez Díaz por su colaboración en la parte estadística.

\section{BIBLIOGRAFIA}

ALIAGA, L. 1990. Manual técnico sobre crianza de lombrices. Lima (Perú): FUNDEAGRO

ARROYO, R. 1984. Curso. Estadística Aplicada a la Investigación Diseños Experimentales. Iquitos (Perú):IIAP

ASCON, G. 1993. Lombricultura una alternativa para el desarrollo de San Martín, Boletín.- "Oportunidades Comerciales"- Cámara de Comercio - Año 1 № 7 - Tarapoto - (San Martín.)

CAMPOVERDE, L. 1977. Aspectos Físicos y Químicos de las quebradas "Ahuashiyacu" (Tarapoto) e "Indañe" (Moyobamba) en el departamento de San Martín. Tesis para optar el grado de Bachiller en CC. BB. Universidad Nacional de Trujillo

FERRUZZI, C. 1987. Manual de Lombricultura. - Ediciones Mundiprensa Madrid (España): Eds. Mundiprensa. 138 pp.

SILVA, F. 1985. Primera Jornada Nacional de Lombricultura Universidad de Santiago de Chile. Escuela Tecnológica. Santiago de Chile. 